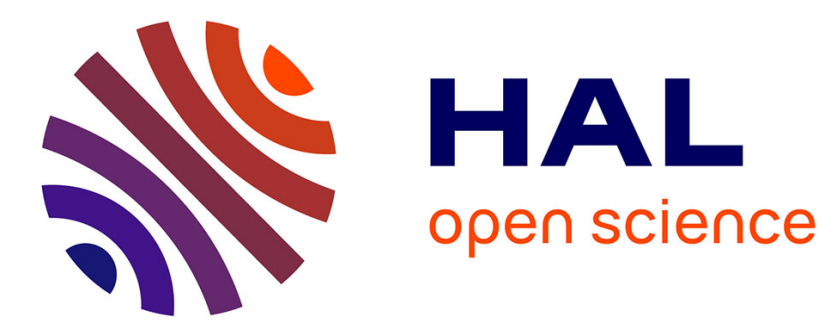

\title{
Claude Perrault (1613-1688), observateur révolutionnaire des animaux
}

Eric Baratay

\section{To cite this version:}

Eric Baratay. Claude Perrault (1613-1688), observateur révolutionnaire des animaux. Dix-septième siècle, 2012, Malebranche et la littérature, 255, pp.309-320. 10.3917/dss.122.0309 . halshs-01569483

\section{HAL Id: halshs-01569483 \\ https://shs.hal.science/halshs-01569483}

Submitted on 27 Jul 2017

HAL is a multi-disciplinary open access archive for the deposit and dissemination of scientific research documents, whether they are published or not. The documents may come from teaching and research institutions in France or abroad, or from public or private research centers.
L'archive ouverte pluridisciplinaire HAL, est destinée au dépôt et à la diffusion de documents scientifiques de niveau recherche, publiés ou non, émanant des établissements d'enseignement et de recherche français ou étrangers, des laboratoires publics ou privés. 


\title{
CLAUDE PERRAULT (1613-1688), OBSERVATEUR RÉVOLUTIONNAIRE DES ANIMAUX
}

\author{
Éric Baratay
}

P.U.F. | Dix-septième siècle

$2012 / 2-n^{\circ} 255$
pages 309 à 320

ISSN 0012-4273

Article disponible en ligne à l'adresse:

http://www.cairn.info/revue-dix-septieme-siecle-2012-2-page-309.htm

Pour citer cet article :

Baratay Éric, « Claude Perrault (1613-1688), observateur révolutionnaire des animaux »,

Dix-septième siècle, 2012/2 n²55, p. 309-320. DOI : 10.3917/dss.122.0309

Distribution électronique Cairn.info pour P.U.F..

(c) P.U.F.. Tous droits réservés pour tous pays.

La reproduction ou représentation de cet article, notamment par photocopie, n'est autorisée que dans les limites des conditions générales d'utilisation du site ou, le cas échéant, des conditions générales de la licence souscrite par votre établissement. Toute autre reproduction ou représentation, en tout ou partie, sous quelque forme et de quelque manière que ce soit, est interdite sauf accord préalable et écrit de l'éditeur, en dehors des cas prévus par la législation en vigueur en France. II est précisé que son stockage dans une base de données est également interdit. 


\section{Claude Perrault (1613-1688), observateur révolutionnaire des animaux}

La profonde "révolution scientifique " du XVII ${ }^{e}$ siècle ne concerne pas que l'astronomie, même si celle-ci est particulièrement illustrée par Kepler, Galilée, Newton, mais toutes les sciences de la nature et en particulier la zoologie qui passe peu à peu d'une configuration ancienne à notre conception moderne entre le milieu du $\mathrm{XVII}^{\mathrm{e}}$ siècle et celui du XVIII ${ }^{\mathrm{e}}$ siècle, de Claude Perrault à Linné. En effet, Perrault ${ }^{1}$ est celui qui initie le plus et le mieux, consciemment et volontairement, une rupture conceptuelle et méthodologique poussée pour fonder une zoologie nouvelle. Né en 1613 dans une famille aisée de la bourgeoisie parisienne, il étudie d'abord la médecine à la faculté de Paris, obtient le grade de docteur en 1641 puis exerce environ 25 ans, mais sans rien publier. Cependant, il semble connaître les travaux majeurs de son époque, notamment ceux d'Harvey ou de Descartes, et il fréquente des assemblées savantes, réunies chez les uns ou les autres sur le modèle des académies romaines et florentines, constituées dans la première moitié du siècle ${ }^{2}$. Sans doute, est-ce cette fréquentation mais aussi, et surtout, la protection de son frère, l'écrivain Charles Perrault, devenu premier commis de la surintendance des bâtiments du roi et conseiller écouté de Colbert en matière de sciences et d'arts, qui lui valent une nomination à l'Académie des sciences dès sa création, en 1666, sur le modèle de la Royal Society de Londres ${ }^{3}$.

1. Sur la biographie et l'œuvre de Claude Perrault, nous renvoyons à l'ouvrage majeur d'Antoine Picon, Claude Perrault, 1613-1688, ou la Curiosité d'un classique, Paris, Picard, 1988 (seconde édition en 2000), notamment p. 13-52 pour la formation de Perrault. L'ouvrage renouvelle une bibliographie datée : André Halleys, Les Perrault, Paris, Perrin, 1931 ; Joseph Lebovits, Claude Perrault physiologiste, Paris, Annette, 1931, thèse de médecine ; Alberto Tenenti, "Claude Perrault et la pensée scientifique française dans la seconde moitié du XVII ${ }^{\mathrm{e}}$ siècle ", L'Éventail de l'histoire vivante. Hommage à Lucien Febvre, Paris, Colin, 1953, II, p. 303-316), mais il est rapide sur le travail zoologique de Perrault, qui n’a jamais bénéficié d'une étude spécifique.

2. Voir Frances Yates, Les Académies en France au XVI siècle, Paris, PuF, 1996 ; Daniel Hurel, Gérard Laudin (éds.), Académies et sociétés savantes en Europe (1650-1800), Paris, Champion, 2001.

3. Arthur Birembaut, "Les caractères originaux de l'Académie royale des sciences de 1666 à 1698 ", Les Sociétés savantes. Leur histoire, Paris, Bibliothèque nationale, 1976, p. 11-13; Roger Hahn, L'Anatomie d'une institution scientifique : l'Académie des sciences de Paris, 1666-1803, Amsterdam, Éditions des archives contemporaines, 1993 ; Margery Purver, The Royal Society : Concept and Creation, London, Routledge, 2008. 


\section{LA CONVERSION À LA ZOOLOGIE}

Cette nomination est l'acte déclencheur d'une carrière scientifique active. À l'Académie, il noue des liens étroits avec le physicien Christian Huygens, le médecin et philosophe Marin Cureau de la Chambre, le mathématicien Gilles de Roberval, le médecin Jean Pecquet, ancien collaborateur d'Harvey et découvreur du canal thoracique, qui stimulent sa réflexion. Surtout, il propose aussitôt un projet scientifique, qu'il fait accepter par l'assemblée et dont il s'empare avec ardeur : bâtir une histoire naturelle sur la liaison active entre le raisonnement et une observation précise, selon le modèle des sciences modernes, notamment de l'astronomie, la discipline phare à l'Académie (ouverture de l'observatoire de Paris en 1671). Claude Perrault propose ainsi de développer une histoire des plantes (on dirait maintenant une botanique) traitant des particularités de culture et des vertus médicinales, mais surtout développant des descriptions précises, des analyses chimiques et physiologiques. À cette fin, un laboratoire de chimie est installé dès 1667 dans une pièce de la nouvelle bibliothèque du roi, rue Vivienne, qui abrite l'Académie, et des expériences sur la circulation de la sève sont effectuées dès $1668^{4}$.

En fait, c'est surtout l'histoire des animaux, autrement dit la zoologie ${ }^{5}$, qui retient l'attention de Claude Perrault, car il bénéficie d'une circonstance exceptionnelle, à l'origine de cette conversion, avec la présence de deux grandes ménageries royales à Vincennes et à Versailles qui devient un pôle scientifique majeur ${ }^{6}$. Or, les ménageries représentent l'une des plus importantes formes de retenue des bêtes sauvages, à côté des parcs et des réserves de chasse, loin devant les modestes installations ambulantes des montreurs ${ }^{7}$. Leur nombre, leur taille, leur diffusion géographique augmentent aux temps modernes, en bonne partie grâce à la multiplication des voyages et aux importations croissantes de bêtes exotiques. Car, l'engouement pour ces animaux rares et curieux ainsi que l'envie de les collectionner s'accroissent à partir du Xvi ${ }^{\mathrm{e}}$ siècle, et c'est d'ailleurs à cette époque qu'apparaît le terme " exotique " dans la littérature française, pour qualifier les objets et les êtres venus des terres lointaines ${ }^{8}$. Cela s'inscrit dans un émerveillement des contemporains pour les œuvres de la

4. Sur ce projet et cette botanique, voir Arthur Birembaut, ibidem, p. 13; Joseph Schiller, "Les laboratoires d'anatomie et de botanique à l'Académie des sciences au XVII ${ }^{e}$ siècle ", Revue d'histoire des sciences, 17, 1964, p. 97-112 ; Yves Laissus, A. M. Monseigny, "Les plantes du roi. Notes sur un ouvrage de botanique préparé au XVII e siècle par l'Académie royale des sciences ", Revue d'histoire des sciences, 22, 1969, p. 193-236.

5. Sur ce sujet délaissé par les historiens: Georges Petit et Jean Théodoridès, Histoire de la zoologie des origines à Linné, Paris, Hermann, 1962 ; Jean-Loup D’Hondt, Histoire de la zoologie, Paris, Ellipses, 2007.

6. Catherine Arminion, Versailles et les Sciences, Paris, Gallimard, 2010 ; Béatrix Saule (dir.), Sciences et curiosités à la cour de Versailles, Paris, RMN, 2010.

7. Éric Baratay, Élisabeth Hardouin-Fugier, Zoos. Histoire des jardins zoologiques en Occident (XVIXXe siècle), Paris, La Découverte, 1998 ; Nigel Rothfels, Savages and Beasts: the Birth of Modern Zoo, Baltimore, Johns Hopkins up, 2002 ; Gérard Mabille, Joan Pieragnoli, La Ménagerie de Versailles, Arles, Honoré Clair, 2010 ; Joan Pieragnoli, « La ménagerie de Versailles (1662-1789) : fonctionnement d'un domaine complexe", Versalia, 13, 2010, p. 173-195.

8. Rabelais, Pantagruel. Quart Livre, chapitre 2, cité par Liliane Bodson (éd.), Les Animaux exotiques dans les relations internationales, Liège, Université de Liège, 1998, p. 145. 
nature, dans un goût prononcé pour les extravagances de sa production, car la nature est perçue comme une entité vivante, disposant d'une certaine autonomie vis-à-vis de Dieu, lui permettant d'inventer, de s'amuser, de décorer le monde en créant des œuvres admirables et singulières?.

Mais ces animaux curieux sont encore rares, aussi précieux et recherchés que le sucre, les épices les plus fines, les pierres ou les fourrures les plus belles. Le coût des achats, peu à peu raisonnable pour une bête avec l'augmentation des importations, reste prohibitif pour un ensemble plus important, d'autant plus que la forte mortalité, due à la médiocrité des logements, oblige à un renouvellement incessant. Les véritables collections sont donc le fait des princes et de la haute aristocratie tandis que les nobles de moindre importance, voire des roturiers, se contentent d'un exemplaire vivant ou de bêtes empaillées ou seulement de parties imputrescibles, bien moins chères et plus aisées à conserver. Parmi les bêtes vivantes, les oiseaux occupent une place prédominante jusqu'au XviII ${ }^{\mathrm{e}}$ siècle : sauvages ou domestiqués, ils séduisent pour la splendeur des chants, des formes ou des plumages, et ils sont érigés en ouvres d'art de la nature. Sont aussi concernées diverses espèces de singes, de carnivores, du lion au guépard, d'herbivores (gazelles, antilopes...) et surtout de bêtes domestiques, plus faciles à transporter et répondant tout autant aux exigences de la curiosité et de la collection : chameaux, dromadaires, lamas, chevaux, moutons, chèvres. En fait, l'éventail des espèces s'élargit peu à peu et l'Europe voit arriver des orangs-outangs en Hollande vers 1640, des crocodiles à Versailles en 1687, des zèbres du Cap aux Pays-Bas ou des kangourous en Angleterre au XVIII ${ }^{\mathrm{e}}$ siècle, etc. Toutes ces espèces exotiques prennent peu à peu le pas (le renversement s'effectue au cours du $\mathrm{XVII}^{\mathrm{e}}$ siècle) sur les animaux indigènes (loup, sanglier, ours, cerf...) qui représentaient l'essentiel des effectifs au Moyen Âge et au Xvi ${ }^{\mathrm{e}}$ siècle ${ }^{10}$.

D'ailleurs, à cette époque, les espèces indigènes et exotiques étaient le plus souvent mélangées, et placées aux abords des demeures aristocratiques. Cependant, des sites réservés aux animaux insolites avaient été créés dans quelques villas italiennes ou sur les terres de seigneurs portugais. Leur nombre augmente au XVII siècle et l'évolution est précipitée par Louis XIV qui fait bâtir un sérail de bêtes "féroces » à Vincennes, en 1661, pour organiser des combats, et une ménagerie d'animaux exotiques, rares et curieux, à Versailles en 1664. La distinction est reprise un peu partout en Europe et les seconds lieux prennent peu à peu ce nom français de « ménagerie ». Issu du terme ménage, évoquant la tenue de la maison, il signifiait l'administration d'une ferme au $\mathrm{XVI}^{\mathrm{e}}$ siècle puis le lieu d'engraissement des bêtes. Il est utilisé des 1664 pour désigner le site des animaux de collection à Versailles, et il acquiert ce sens d'espace réservé aux animaux étrangers et curieux, près des châteaux, dans les dictionnaires de la fin du siècle ${ }^{11}$.

9. Voir Jean Céard, La Nature et les prodiges, l'insolite au XVI siècle, Genève, Droz, 1977.

10. Pour un panorama historique de la condition animale, voir Éric Baratay, Et l'homme créa l'animal. Histoire d'une condition, Paris, Odile Jacob, 2003 ; Linda Kalof, Brigitte Resl (dir.), A Cultural History of Animals, Oxford, Berg Publishers, 2007. Pour la période : Philip Ford (éd.), L'Animal sauvage à la Renaissance, Cambridge, French Colloquia, 2007 ; Charles Mazoyer (éd.), L’Animal au XVII siècle, Tübingen, Narr, 2003.

11. Voir les articles "Ménagerie " des dictionnaires de l'Académie française (1684), de Furetière (1690) et de Trévoux (1721). 
Jusqu’à la première moitié du XVII ${ }^{\mathrm{e}}$ siècle, fauves, éléphant, rhinocéros, etc. servaient aux parades, pour affirmer le faste, la puissance, la domination du propriétaire, mais aussi la parenté de celui-ci avec le sauvage, avec qui il partagerait force, fougue et bravoure. Les combats, en usage jusqu'à la fin du siècle, opposant le plus souvent des bêtes sauvages captives à des domestiques (chiens, bovins...), exaltent la prodigalité et le statut extraordinaire du maître qui sacrifie des biens précieux pour le divertissement et le plaisir de l'entourage. Les collections vivantes, dépourvues d'usages concrets, au statut proche des œuvres d'art, expriment le goût du gratuit, le dédain de l'utilité vulgaire, renforcent la distance avec le reste de la société, toujours contraint par les contingences matérielles et la nécessité. La progressive concentration sur les animaux exotiques, curieux et rares, permet de souligner le prix énorme de la collection et l'effort entrepris par le propriétaire. Outre ces affirmations essentielles d'un statut extraordinaire du maître, la possession des bêtes exalte aussi son degré de culture, exprimé d'une autre manière par les collections de spécimens, présentées dans les cabinets de curiosité, de plantes rares, disposées dans les jardins botaniques, et d'œuvres d'art, que de nombreuses demeures laissent admirer ensemble, au long de circuits organisés, comme à la villa Borghèse à Rome, ou à Versailles. Enfin, la détention des bêtes permet de participer à l'effort de connaissance du monde, et l'œuvre de Claude Perrault s'inscrit dans ce cadre.

\section{OBSERVER SOI-MÊME DE VRAIS ANIMAUX}

Elle repose sur la conviction qu'il est impossible de laisser le travail d'observation aux voyageurs contemporains, accusés de peu et mal regarder, d'être "dépourvus de science et de patience ", trop attirés par l'extraordinaire aux dépens de l'ordinaire, trop tentés d'amplifier et de déformer pour attirer l'attention, trop prompts à prendre dans les livres ce qu'ils n'ont pas vu, et ainsi de véhiculer les erreurs ${ }^{12}$. L'un des premiers, Claude Perrault transforme en principe une méfiance qui était née parmi les naturalistes du XVI ${ }^{\mathrm{e}}$ siècle, avec les récits extraordinaires des découvertes, et qui se maintient jusqu'au début du $\mathrm{Xx}^{\mathrm{e}}$ siècle $^{13}$. Devant en conséquence observer par eux-mêmes, les naturalistes ont l'obligation de surmonter l'indigence des moyens habituels. Car la rareté et la cherté des bêtes exotiques obligent longtemps les savants à faire feu de tout bois, à utiliser notamment toutes les reproductions possibles : statuettes, monnaies et surtout dessins ou gravures. L'image joue un rôle fondamental pour diffuser les savoirs et favoriser la réflexion, car elle permet de voir, donc de décrire, de nommer et de classer. Mais elle souffre de deux handicaps importants aux $\mathrm{XVI}^{\mathrm{e}}$-XVIII ${ }^{\mathrm{e}}$ siècles : le coût d'impression freine la publication de recueils nouveaux ; par économie, les libraires préferrent souvent rééditer de vieilles gravures ou se contentent de dessins médiocres, reproduisant et confortant les erreurs ${ }^{14}$.

12. Mémoires pour servir à l'histoire naturelle des animaux, Paris, Imprimerie royale, 1671-1676, I, préface non paginée.

13. Éric Baratay, Portraits d'animaux. Les planches du Dictionnaire universel d'histoire naturelle de Charles d'Orbigny (1841-1849), Lyon, Fage, 2007.

14. Sur le rôle et le statut de l'image en histoire naturelle, voir Madeleine Pinault (éd.), Dessin et science, XVIT'-XVIII siècles, Paris, Réunion des musées nationaux, 1984, et Le Peintre et l'Histoire naturelle, Paris, Flammarion, 1990, p. 31-34, 179-180 ; Éric Baratay, op. cit. 
Pour Perrault, les naturalistes ne peuvent pas, non plus, s'en tenir aux seuls animaux tués au loin puis expédiés en France, dont les os, les peaux, voire les dépouilles naturalisées s’acquièrent assez aisément. Ces éléments sont préservés par salage temporaire, réduction au squelette ou à la peau, taxidermie rudimentaire avec bourrage à la paille. À partir du XviI ${ }^{\mathrm{e}}$ siècle, l'utilisation du savon arsenical, qui désinfecte les peaux, les plumes et les fourrures, de l'esprit-de-vin ou de l'eau-de-vie permet même de conserver l'animal entier. Cependant, les inconvénients sont de plus en plus soulignés à mesure que l'exigence d'exactitude s'accroît. Les fourrures et les parties molles sont souvent décomposées ou mangées par les insectes et les rongeurs, ou encore racornies par les conservateurs. Les peaux s'allongent, faussent les mesures et les formes, sont difficiles à remonter. Dès lors, les spécimens sont souvent mal reconstitués, déformés, incomplets soit parce que les organes ont été enlevés, soit parce qu'ils sont détériorés, tel ce castor d'Amérique étudié par Perrault, dont les yeux, mangés par les rats, ne peuvent être examinés ${ }^{15}$.

D'où la volonté de travailler majoritairement sur des animaux vivants ou venant de décéder et encore intacts. Mais cela suppose des sources d'approvisionnement fiables, assez faciles à établir pour les espèces indigènes, les poissons et les mammifères marins des eaux européennes, avec les dons de particuliers, beaucoup plus difficiles à installer pour les bêtes exotiques et, là encore, les naturalistes de l'époque usent de toutes les possibilités. Ils se précipitent notamment dans les foires pour observer les bêtes des montreurs. En 1631, par exemple, Peiresc va d'Aix à Toulon à pied pour observer l'éléphant Hansken, alors célèbre dans toute l'Europe qu'il traverse peu à peu : «Il vint passer par ici, où il fut trois jours, durant lesquels je le considérai bien à mon aise et avec grand plaisir, ne l'ayant pas laissé échapper de mes mains ou dépayser que je ne l'aie fait peser, contre six vingt boulets de canon. Il me connaissait déjà quasi comme son gouverneur, et je me laissais porter jusqu'à ce point de curiosité ou (pour mieux dire) de folie, que de lui mettre ma main dans sa bouche et de lui manier et empoigner une de ses dents maxillaires pour en mieux reconnaître la forme, et ne les ayant pas assez bien pu voir sans les toucher, à cause qu'en ouvrant la gueule, il les entrecouvrait avec sa langue ${ }^{16}$. "Le témoignage montre bien les limites du recours, les observations étant forcément rapides et cantonnées à quelques aspects. La possession de bêtes est une autre solution, mais son coût la réduit à de rares exemplaires et si cela semblait merveilleux au XVI ${ }^{\mathrm{e}}$ siècle, encore suffisant dans la première moitié du $\mathrm{XVII}^{\mathrm{e}}$ siècle, cela devient dérisoire à l'époque de Perrault.

De grands établissements paraissent de plus en plus indispensables, comme l'a suggéré Francis Bacon dans La Nouvelle Atlantide (1627) en présentant une cité scientifique idéale, où des enclos et des bassins renfermeraient diverses espèces pour des opérations de vivisection et de dissection, de croisement et de modification des allures $^{17}$. Tout cela incite les naturalistes à solliciter le recours aux ménageries des princes et des plus grands aristocrates, comme ils le font aussi pour leurs cabinets de curiosité et

15. Description anatomique de divers animaux disséqués dans l'Académie royale des sciences, Paris, D'Houry, 1682, p. 53.

16. Pierre Tamisey de Laroque (éd.), Lettres de Peiresc aux frères Dupuy, Paris, Imprimerie nationale, 1890, t. II, p. 293-294.

17. Paris, Payot, 1983 , p. $75-76$. 
leurs jardins botaniques dont ils obtiennent souvent les directions. Les propriétaires des ménageries vont rarement jusqu'à ce point, car le coût et l'exception de leur collection vivante en font un attribut inégalé qu'ils ne veulent pas partager, mais ils ouvrent volontiers les portes aux savants pour pouvoir s'affirmer protecteurs des sciences.

Ainsi, à partir de 1667, Claude Perrault multiplie les descriptions morphologiques et les études anatomiques, au moyen de dissections, à la ménagerie de Versailles mais aussi à la bibliothèque du roi et au jardin du roi, où l'on amène les bêtes des ménageries de Vincennes et Versailles. Les ouvrages de Perrault, les registres de l'Académie des sciences, les comptes des bâtiments du roi ${ }^{18}$ montrent bien l'ampleur de la mobilisation de la faune royale (lion, éléphant, gazelle, caméléon, ours, crocodile, dromadaire, castor, civette, élan, etc.), notamment de la ménagerie des animaux « féroces » de Vincennes et de celle des espèces rares et curieuses de Versailles, qui trouvent là une fonction scientifique d'importance. Il s'agit de construire une histoire naturelle digne de Louis XIV, que Perrault place au-dessus d'Alexandre alors que lui-même s'imaginerait bien en nouvel Aristote, celui-ci ayant justement eu la permission d'étudier dans la ménagerie de l'empereur antique ${ }^{19}$. Aussi le soutien royal est-il manifeste; il culmine avec la présence du monarque à la dissection de l'éléphant de Versailles, mort en $1681:$ : [...] le roi ne dédaigna pas d'être présent à l'examen de quelques-unes des parties ; et lorsqu'il y entra, il demanda avec empressement où était l'anatomiste qu'il ne voyait point; M. du Verney s'éleva aussitôt des flancs de l'animal, où il était, pour ainsi dire, englouti. ${ }^{20} \mathrm{C}^{\prime}$ est pour rendre compte de ses observations dans un cadre aussi propice que Claude Perrault publie ses premières œuvres scientifiques : d'abord deux opuscules en 1667 et $1669^{21}$, dont le second, intitulé Description anatomique d'un caméléon, d'un castor, d'un dromadaire, d'un ours et d'une gazelle, est souvent considéré comme la première publication officielle de l'Académie des sciences, et surtout les Mémoires pour servir à l'histoire naturelle des animaux, qui paraissent en deux étapes, en 1671 et $1676^{22}$, et qui représentent l'œuvre phare de la Compagnie en cette première décennie d'existence $^{23}$. Claude Perrault profite de ces publications pour exposer sa problématique, sa méthode, ses résultats, et surtout pour exprimer sa volonté de rompre avec une

18. Jules Guiffrey, Comptes des bâtiments du roi sous le règne de Louis XIV, Paris, Imprimerie nationale, 1881-1886, I, p. 448, 565, 993, II, p. 102, 536 ; Mémoires, op. cit., p. 16, 157, 221.

19. Mémoires pour servir à l'Histoire naturelle des animaux, Paris, Imprimerie royale, 1671, préface n. p.

20. Bernard de Fontenelle, Histoire de l'Académie royale des sciences, Paris, Martin, 1733, I, p. 322.

21. Extrait d'une lettre écrite à $M$. de La Chambre qui contient les observations qui ont été faites sur un grand poisson disséqué à la Bibliothèque du Roy, le 24 juin 1667. Observations qui ont été faites sur un lion disséqué dans la Bibliothèque du Roy, le 28 juin 1667, tirées d'une lettre à $M$. de La Chambre, Paris, Léonard, 1667 ; Description anatomique d'un caméléon, d'un castor, d'un dromadaire, d'un ours et d'une gazelle, Paris, Léonard, 1669, repris dans Description anatomique de divers animaux disséqués dans l'Académie royale des sciences, Paris, D'Houry, 1682.

22. Paris, Imprimerie royale, 1671, 91 pages, et 1671-1676, deux volumes.

23. Sur la mise en place à l'époque d'une communication savante européenne : Jean-Pierre Vittu, "Les journaux savants formes de la communication et agents de la construction des savoirs $\left(17^{\mathrm{e}}-18^{\mathrm{e}}\right.$ siècles)", Dix-huitieme siècle, 40, 2008, p. 281-300. 
zoologie plus livresque qu'expérimentale, plus portée sur la référence aux autorités antiques et sur la compilation que sur l'observation.

\section{UNE RUPTURE ÉPISTÉMOLOGIQUE}

Car ce sont bien les animaux des ménageries royales qui permettent à Perrault de rompre avec les prédécesseurs accusés de compiler sans vérifier, de véhiculer les erreurs, de se soumettre à l'autorité des Anciens comme des " aveugles incapables de voir et de distinguer le vrai du faux ${ }^{24}$. En cela, Perrault se place en tête d'une tendance profonde de l'histoire naturelle à l'époque moderne, c'est-à-dire une confrontation croissante entre la réalité des bêtes et un savoir livresque, issu de compilations successives, qui faisait autorité. En effet, depuis le début du Xvi siècle, les auteurs de l'Antiquité, notamment Aristote et Pline l'Ancien, étaient bien plus lus et connus qu'autrefois, grâce à l'imprimerie. Leur autorité était aussi renforcée par la croyance en un âge d'or situé dans le passé, qui faisait croire qu'ils avaient tout dit et l'avaient bien $\operatorname{dit}^{25}$. Or, les naturalistes découvrent peu à peu que ces anciens n'ont pas connu nombre d'espèces nouvellement arrivées en Europe (qu'il faut donc observer avec un regard vide de référence) et surtout qu'ils se sont souvent trompés parce qu'ils ne disposaient pas des bêtes auprès d'eux. Schématiquement, on peut dire que les naturalistes sont d'abord allés dans les ménageries pour voir les animaux décrits par les anciens et mieux comprendre les textes de ces derniers (ceci est très net au $\mathrm{XVI}^{\mathrm{e}}$ siècle), puis, sans continuité linéaire, d'une manière chaotique selon les pays et les savants entre $\mathrm{XVI}^{\mathrm{e}}$ et XVII ${ }^{\mathrm{e}}$ siècles, pour vérifier, corriger, compléter ces textes, enfin pour observer par soi-même et remplacer des connaissances livresques jugées de plus en plus défaillantes. Cette dernière attitude ne se développe vraiment que dans la seconde moitié du XVII ${ }^{\mathrm{e}}$ siècle sous l'influence de la révolution scientifique ou, plus précisément, de la révolution cartésienne. On l'appelle souvent ainsi par commodité, parce qu'elle a été théorisée par Francis Bacon et surtout par Descartes dans son Discours de la méthode pour bien conduire sa raison et chercher la vérité dans les sciences (1637), mais elle était en réalité portée par nombre d'esprits cultivés dans la première moitié du XVII ${ }^{\mathrm{e}}$ siècle. Elle consiste à douter et à faire table rase des connaissances héritées, à observer et raisonner par soi-même, à construire un savoir nouveau, par étapes progressives, prouvées, vérifiées l'une après l'autre, dans une recherche incessante de la vérité.

Portant cela très haut, Claude Perrault prône l'observation précise des faits, "sans autre intention que de faire voir les choses telles que nous les avons vues de même qu'en un miroir qui ne met rien du sien ». Cette foi dans la neutralité du regard, considéré comme la seule solution pour lutter contre l'autorité des Anciens, est aussi bien issue des observations de Galilée, consignées dans Le Messager des étoiles de 1610 (au titre significatif de cet état d'esprit), que des préceptes de Descartes. Elle

24. Mémoires, op. cit. (seconde édition), préface n. p.

25. Charles Schmitt, Aristote et la Renaissance, Paris, Puf, 1992 ; Platon et Aristote à la Renaissance, Paris, Vrin, 1976 ; Luca Bianchi « "Interpréter Aristote par Aristote". Parcours de l'herméneutique philosophique à la Renaissance ", Methodos, 2, 2002, en ligne sur revues.org 
suppose deux corollaires qui forment les autres éléments de la rupture épistémologique. L'observation doit être contrôlée, vérifiée pour être prouvée. Sa description, par le texte et le dessin, doit être une "peinture naïve, avec simplicité, sans ornement ", dégagée de toute interprétation théorique et de tout effet littéraire ou artistique $^{26}$. À cette fin, Perrault installe deux procédures. Il organise un travail en équipe avec des médecins anatomistes, tels Gayant, Pecquet ou Duverney, qui mesurent et dissèquent, des artistes, tels La Hire, Le Clerc, qui dessinent ; lui-même rédige les comptes rendus. Rédactions et dessins sont effectués au moment des opérations en concertation entre les participants. Ils sont ensuite relus ou regardés, vérifiés, approuvés par l'équipe puis par l'assemblée de l'Académie ${ }^{27}$. L'autre procédure, développée au même moment en Angleterre, consiste à inviter des observateurs, qui deviennent les témoins et les garants de la réalité de l'observation et de l'exactitude des comptes rendus. Ce sont souvent des savants mais aussi des aristocrates dont le rang social fait que leurs témoignages ne peuvent être mis en doute. L'exemple le plus célèbre est encore cette dissection de l'éléphant, marquée, d'après Fontenelle, par « la qualité et le nombre des assistants $»^{28}$, dont le roi lui-même.

De tout cela découlent deux aspects méthodologiques. D'abord une modestie revendiquée : il s'agit moins de trouver des choses nouvelles que d'acquérir des certitudes sur les faits observés. Ces certitudes, même modestes, sont plus importantes que les affirmations hasardeuses ou que les interprétations, qu'il préfere repousser à plus tard ou seulement suggérer à titre de simples hypothèses ${ }^{29}$. L'autre aspect est la prudence devant guider la construction de la science nouvelle et la présentation des résultats. Perrault affirme, par exemple, ne pas avoir étudié le lion, l'éléphant ou l'ours, en tant qu'espèces, mais un lion, un éléphant, un ours. L'extrapolation de l'individu à l'espèce ne peut être faite qu'à la suite d'observations ou de témoignages concordants. Ainsi, Perrault invite un voyageur arabe à assister à la dissection d'un chameau de Versailles pour qu'il lui certifie que les chameaux de son pays sont semblables à celui$\mathrm{ci}^{30}$. Ces principes se retrouvent dans les publications de Perrault, dont les formes et les titres proclament leur qualité de précises et modestes monographies, et non plus d'encyclopédies compilant tout ce qui a été écrit sur tous les aspects, comme l'est encore celle de Jan Jonston, la dernière du genre, parue entre 1657 et $1667^{31}$. En cela, Perrault s'inscrit totalement dans la «révolution scientifique» du siècle ${ }^{32}$,

26. Mémoires, op. cit. (seconde édition), préface n. p.

27. Voir Joseph Schiller, art. cit., p. 104, et Alberto Tenenti, art. cit., p. 304. Sur la révolution des instruments : Philippe Hammou, La Mutation du visible. Essai sur la portée épistémologique des instruments au XVII siècle, Villeneuve-d'Asq, Septentrion, 1999-2001.

28. Op. cit., I, p. 322. Sur cette présence des aristocrates dans les expériences savantes au XvII siècle, voir Christian Licoppe, La Formation de la pratique scientifique : le discours de l'expérience en France et en Angleterre, Paris, La Découverte, 1996. Sur la méthode scientifique : Claire Salomon-Bayet, L'Institution de la science et l'expérience du vivant. Méthode et expérience à l'Académie royale des sciences, 1666-1793, Paris, Flammarion, 1978.

29. "Les réflexions inscrites ne sont pas des décisions mais seulement des essais", Mémoires, op. cit., (seconde édition), préface n. p.

30. Mémoires, ibid., préface n. p.; Description, op. cit., p. 74.

31. Historie naturalis, Amsterdam, Schipper.

32. Marie Boas Hall, Promoting Experimental Learning : Experiment and the Royal Society, 1660-1727, Cambridge, Cambridge University Press, 1991. 
qui ne concerne pas seulement les sciences de la nature, mais nombre de disciplines comme l'histoire. Ainsi, le titre et la problématique de l'ouvrage majeur de LouisSébastien Le Nain De Tillemont (Mémoires pour servir à l'histoire ecclésiastique des six premiers siècles) résonnent étroitement avec ceux de Perrault ${ }^{33}$.

\section{DÉCRIRE LES CORPS, NÉGLIGER LES COMPORTEMENTS}

Mais à quoi s'intéresse-t-il lorsqu'il observe les bêtes et notamment les espèces rares des ménageries? Ne voulant s'appuyer que sur ce qu'il voit, il s'attache, pour les animaux vivants, à "bien dépeindre leur forme extérieure et à marquer la grandeur et la proportion de toutes les parties qui se voient sans dissection " ${ }^{34}$. Ainsi, l'étude du caméléon se concrétise par une description, voire une mensuration précises des membres et de la peau, des mouvements des yeux, de la tête, de la mâchoire, des pattes, de la queue et du cour, des variations de grosseur et de couleur selon les moments et les lieux, des manières de marcher, de saisir les objets et de manger. À propos de la couleur, il organise de multiples expériences, plaçant l'animal au soleil, à l'ombre, dans un linge blanc, sur des choses et des gens pour mesurer l'intensité et la durée des variations ${ }^{35}$. Les animaux décédés donnent lieu aux mêmes descriptions et mensurations des membres, mais surtout font l'objet de dissections pour décrire les organes et les squelettes. Plus que la morphologie, l'anatomie est au cœur de l'entreprise de Perrault, car il considère, à juste titre, que c'est l'aspect le moins connu de l'histoire des animaux. L'étude anatomique permet de montrer le parallèle entre les membres et les organes de la même bête, mais aussi les analogies de forme entre les vivants. Cette idée d'une structure commune, qu'on retrouve dans la dissection de l'éléphant, censée dévoiler des aspects anatomiques encore obscurs chez l'homme ${ }^{36}$, permet d'esquisser une anatomie comparée, qui devient ensuite un élément majeur de l'histoire naturelle au XVIII ${ }^{\mathrm{e}}$ siècle. Que ce soit pour la morphologie ou l'anatomie, la transcription des observations en ordres de grandeur, de poids, de forme ${ }^{37}$, montre l'imposition sur les vivants d'une grille de lecture mathématique et géométrique, comme Harvey l'avait fait pour la circulation du sang, Kepler et Galilée pour les planètes, et comme Descartes l'avait prôné.

Pour chaque animal étudié, Perrault résume, discute et contredit souvent les affirmations des prédécesseurs, essentiellement les auteurs de l'Antiquité et les encyclopédistes des $\mathrm{XVI}^{\mathrm{e}}-\mathrm{XVII}^{\mathrm{e}}$ siècles. Là encore, il organise des expériences pour prouver leurs erreurs. Il met le caméléon de Versailles en présence d'un corbeau pour montrer que ce dernier ne meurt pas des quelques coups de bec qu'il porte au premier,

33. Paris, Robustel, 1694-1712. Voir Bruno Neveu, "Sébastien Le Nain de Tillemont, 16371698, et l'érudition ecclésiastique de son temps ", Religion, érudition et critique à la fin du XVII siècle et au début du XVIIt siècle, Paris, PUF, 1968, p. 21-32 ; S. M. Pellistrandi (éd.), Le Nain de Tillemont et l'historiographie de l'Antiquité tardive, Paris, Champion, 2002.

34. Mémoires, op. cit. (seconde édition), préface n. p.

35. Description, op. cit., p. 5-20.

36. Registres manuscrits de l'Académie des sciences, février 1681, folio 82, cités par Joseph Schiller, art. cit., p. 109.

37. Description., op. cit., p. 92-100. 
contrairement à ce que Solin avait écrit. Il place le même caméléon sur un figuier sauvage et constate qu'il reste tranquille et froid alors que Pline l'Ancien avait affirmé que ce contact l'échauffait et le rendait furieux ${ }^{38}$. À l'inverse, Perrault fait exécuter de nombreux dessins, qui prennent ainsi un statut scientifique de premier plan, supérieur au texte (et maintenu jusqu'à la fin du $\mathrm{XIX}^{\mathrm{e}}$ siècle et le remplacement du crayon par l'appareil photographique), pour montrer et prouver ce qui a été observé et décrit, que ce soient les mouvements d'un vivant, ou la structure d'un mort, tel l'ours représenté deux fois, avec et sans peau ${ }^{39}$. En réalité, les animaux étudiés après leur décès sont bien plus nombreux que les vivants, et le travail de Perrault illustre ce partage des bêtes des ménageries, qui s'effectue à l'époque et qui dure jusqu'au $\mathrm{XIX}^{\mathrm{e}}$ siècle : aux princes, aux grands aristocrates, mais aussi aux courtisans et au public, les animaux vivants contemplés dans leurs cages ou leurs enclos ; aux naturalistes, les animaux décédés, concédés pour le bien de la science et disséqués.

Il en résulte assez peu d'observations sur les comportements individuels et sociaux. Là encore, Perrault rompt avec les usages en ne reprenant pas les multiples affirmations et suppositions sur les mœurs et les comportements, qui servaient souvent, auparavant, à ériger des exemples ou à développer des morales, sans qu'on puisse et qu'on veuille vérifier la véracité des dires. Ce refus de Perrault s'appuie en bonne partie sur les conditions de vie des animaux dans les ménageries, en particulier à Versailles ou Vincennes. Elles détiennent le plus souvent des spécimens individuels, rarement des couples et encore moins des groupes, dans des situations artificielles et précaires, ne permettant pas de voir la réalité des gestes et des mœurs. Perrault en est bien conscient lorsqu'il note qu'un castor est resté enfermé plusieurs années sans pouvoir aller dans l'eau (à tel point qu'il se demande si ce n'est pas pour cela qu'il ne trouve pas, lors de la dissection, le trou de Botalle assuré par plusieurs auteurs; il se serait peut-être bouché !), ou en décrivant le dépérissement d'un lion qu'on ne sait pas nourrir : " [...] avant qu'il soit mort, il fit plusieurs mois sans vouloir sortir de sa loge, et que l'on avait de la peine à le faire manger ; que pour cela on lui ordonna quelques remèdes et entre autres de ne manger que des chairs de jeunes animaux et de les lui donner vivants. Mais que ceux qui gouvernent les bêtes du parc de Vincennes, pour rendre cette nourriture plus délicate, ajoutèrent une préparation assez extraordinaire. C'est qu'ils écorchaient les agneaux tout vifs et qu'ils lui en firent ainsi manger plusieurs, ce qui d'abord le remit, en lui rendant l'appétit et quelque gaieté. Mais il y a apparence que cette nourriture engendra trop de sang, et qui était trop subtil pour un animal à qui la nature n'a pas donné l'industrie d'écorcher ceux qu'il mange ; étant croyable que le poil, la laine, les plumes et les écailles que tous les animaux de proie avalent sont un assaisonnement et un correctif nécessaires pour empêcher que leur avidité ne les fasse emplir d'une nourriture trop succulente. " ${ }^{40}$

Lautre aspect incitant Perrault à négliger les comportements est son adhésion au cartésianisme. En 1680, il publie La Mécanique des animaux dans ses Essais de

38. Ibidem, p. 47-48.

39. Ibid., p. 49, 71, 83, 104 ; Mémoires, op. cit., p. 191. Voir Joseph Schiller, «L'iconographie de Claude Perrault (1613-1688) ", XC congrès des sociétés savantes, Paris, Bibliothèque Nationale, 1967, I, p. $215-234$.

40. Description, ibid., p. 68, 16 (citation). 
physique $^{41}$. Ce petit traité représente une réflexion théorique à partir des résultats et des hypothèses issus des observations sur les bêtes. Perrault accepte le concept cartésien de l'animal-machine tout en l'amendant, car ses études, qui montrent là toute leur importance, le persuadent des limites de l'explication : les bêtes sont plus compliquées anatomiquement que les horloges et les automates; leurs mouvements sont souvent imprévisibles ; donc un principe, qu'il qualifie d' "âme ", doit activer la machine qui serait inerte sans cela. Il reste que, même corrigé (à l'instar de ce que font beaucoup de cartésiens à cette époque), ce cartésianisme explique l'intérêt quasi exclusif de Perrault pour la morphologie et surtout pour l'anatomie et la physiologie, qui permettent de connaître les rouages de la machine, et son oubli de comportements mal explicables, peut-être redevables d'une âme dont l'étude relève, de l'avis de tous, des philosophes et non des naturalistes ${ }^{42}$.

En fait, comme la plupart de ses contemporains lettrés, Perrault éprouve une forte attirance pour le permanent, le régulier, le mécanique et, au contraire, un désintérêt pour le mouvant, le changeant, l'éphémère, l'imprévisible. Cela se voit bien dans ses autres préoccupations, car il se veut un esprit universel. Il prend grand intérêt à la physiologie et notamment, dans le sillage d'Harvey ${ }^{43}$, à la question des moteurs des machines vivantes, les circulations vitales (sève ou sang) et le cerveau; ce qui le conduit à pratiquer la vivisection de chiens ou de chevaux avec son équipe ${ }^{44}$. Il s'intéresse à la physique mécanique, comme toute son époque, celle des astres mais aussi celle des machines, surtout les horloges et les automates ${ }^{45}$, qui représente d'ailleurs le troisième secteur de recherche développé par la nouvelle Académie des sciences, à côté de l'astronomie et de l'histoire naturelle. Enfin, il se passionne pour l'architecture, aussi bien théorique que pratique. Il traduit Vitruve, s'investit dans la querelle des Anciens et des Modernes, élabore une théorie des ordres des colonnes ${ }^{46}$. Il participe au projet et à la construction de la colonnade du Louvre entre 1667 et 1670,

41. Paris, Coignard, 1680-1688, vol. III. Sur cela, Alberto Tenenti, art. cit., II, p. 307-311; François Azouvi, «Entre Descartes et Leibniz : l'animisme dans les Essais de Physique de Claude Perrault ", Recherches sur le XVII siècle, 5, 1982, p. 9-19.

42. Sur le cartésianisme zoologique et son accueil : Thierry Gonthier, De l'animal à l'homme. Montaigne et Descartes ou les Paradoxes de la philosophie moderne sur la nature des animaux, Paris, Vrin, 1998, et (dir.), Animal et animalité dans la philosophie de la Renaissance et de l'âge classique, Louvain, Peeters, 2005 ; Éric Baratay, L'Église et l'Animal (XVII'-XX' siècle), Paris, Le Cerf, 1996 ; Jean-Luc Guichet, (dir.), De l'animal machine à l'âme des machines, querelles biomécaniques de l'âme (XVII-XXI'), Paris, $\mathrm{PU}$ Sorbonne, 2010.

43. Sur Harvey et son héritage au XvII ${ }^{\mathrm{e}}$ siècle: Gweneth Whitteridge, William Harvey and the Circulation on the Blood, Elsevier Science, 1971; Roger French, William Harvey's Natural Philosophy, Cambridge, University Press, 2008.

44. François Laplossotte, "Quelques étapes de la physiologie du cerveau du XviI ${ }^{\mathrm{e}}$ au début du XIX ${ }^{e}$ siècle ", Annales ESC, 25, 1970, p. 608 ; Joseph Schiller, "Les laboratoires ", art. cit., p. 101, 105.

45. Recueil de plusieurs machines de nouvelle invention, Paris, Coignard, 1700. Sur la curiosité pour les machines: Anthony Turner, "Grollière de Serière, les frères Monconys : curiosité et collecte au XVII ${ }^{e}$ siècle ", La Passion de la collecte : aux origines du musée des Confluences, Lyon, Département du Rhône, 2008, p. 17-27.

46. Les Dix Livres d'architecture (1673), Paris, Libraires associés, 1965 ; Ordonnance des cinq espèces de colonnes selon la méthode des Anciens, Paris, Coignard, 1683. Voir Nathalie Heinlich, "Arts et sciences à l'âge classique ", Actes de la recherche en sciences sociales, 66, 1986, p. 47-78; Wolfgang Hermann, La Théorie de Claude Perrault, Bruxelles, Mardaga, 1995. 
et il exécute les plans de l'observatoire de Paris la même année $1667^{47}$. Et c'est à lui qu'en 1672 Louis XIV dicte personnellement l'Ordre à observer pour les fontaines de Versailles pour contraindre l'eau, concentrée dans les tuyaux et métamorphosée en cristal liquide par les jets d'eau, à dessiner des figures géométriques en des successions voulues $^{48}$. Tout cela montre le goût de Claude Perrault pour une lecture mathématique et géométrique du réel.

Il meurt en 1688 d'une infection provoquée par une piqûre accidentelle avec un instrument lors de la dissection, au jardin du Roi, d'un chameau de la ménagerie de Versailles, dans des conditions d'hygiène évidemment réduites. Son décès signe l'arrêt, pour un temps, des publications en histoire naturelle à l'Académie des sciences. Cependant, son programme est à la base de la zoologie des XVIII et XIX ${ }^{e}$ siècles, notamment cette nécessité d'observer par soi-même et ce recours privilégié aux animaux des ménageries, vivants et décédés. Il reste qu'il ne s'est intéressé ni à la classification des espèces, qui devient la grande affaire des naturalistes à la suite de John Ray et de Linné, ni aux comportements, qu'un Buffon remet à l'honneur en laissant à Daubenton le genre de travail initié par Perrault. Et parce que Buffon est persuadé, lui aussi, que la captivité dans les ménageries déforme et transforme les attitudes animales, il recourt souvent aux textes des Anciens et des voyageurs ${ }^{49}$, que Perrault avait honnis !

Éric BaRATAY

Université Jean-Moulin Lyon-III

47. Antoine Picon, op. cit., p. 103 sq., et Michael Petzet, Claude Perrault und die Architektur der Sonnenkönigs, Munchen, Deutscher Kunstverlag, 2000.

48. Archives nationales, $\mathrm{O}^{1} 1854 \mathrm{n}^{\circ} 4$, dicté par le roi le 18 août 1672 ; voir Éric Souillard, "Les eaux de Versailles sous Louis XIV ", Hypothèses, 1, 1997, p. 105-112 ; Gérard Mabille, Jacques Givry, Jeux d'eaux à Versailles, Paris, Naïve, 2009.

49. Jacques Roger, Buffon, Paris, Fayard, 1989 ; Martin Wåhlberg, "Littérature de voyage et savoir : la méthode de lecture de Buffon ", Dix-huitième siècle, 42, 2010, p. 599-616. 